\title{
Necessity of an exact anatomical understanding for the better pain practice
}

\author{
Yeon-Dong Kim ${ }^{1,2}$ \\ 'Department of Anesthesiology and Pain Medicine, Wonkwang University School of Medicine, Iksan, Korea \\ 'Jesaeng-Euise Clinical Anatomy Center, Wonkwang University School of Medicine, Iksan, Korea
}

Received September 2, 2021, Revised September 5, 2021, Accepted September 6, 2021

Handling Editor: Francis S. Nahm

Correspondence

Yeon-Dong Kim

Department of Anesthesiology and Pain Medicine, Wonkwang University School of Medicine, Wonkwang University Hospital, 895 Muwang-ro, lksan 54538 , Korea

Tel: +82-63-859-1562, Fax: +82-63-857-5472, E-mail: kydpain@wku.ac.kr

Pain medicine has evolved into a subspecialty of its own, separate from anesthesia, and is defined as "the discipline of medicine devoted to the diagnosis and treatment of pain-related disorders, principally with the application of interventional techniques in managing acute, chronic, persistent, and intractable pain, independently" [1]. It continues to develop as a medical field in the spotlight.

Moreover, the unprecedented development and progress in the management of pain, specifically utilizing these interventional techniques, heralded the evolution of interventional pain management cited by or through the Korean Journal of Pain (KJP). Of particular interest are researches that re-examine the anatomical structures that may be meaningful in relation to the cause of pain or its treatment [2-5].

A good example is research that examines exact anatomical locations with the current status of clinical practice, such as the study on the positions of stellate ganglion [6]. In the past, research on the location of the cervical sympathetic ganglion was published which categorized it as a structure of little significance to anatomists [7]. However, the development of new techniques and devices is re- emphasizing its clinical significance by presenting new injection target points, different from those previously known $[8,9]$.

The authors, each with more than 20 years of experience in the field of pain medicine or human anatomy, discovered interesting facts in their conversation [4]. They found differences in the perceptions of the clinical significance of a particular anatomical structure, and came to the conclusion that these differences were more common than they had previously thought. There are definite differences between the anatomical structures found in the textbooks and what is actually encountered in clinical practice. The beginnings of this difference are found in a major reference textbook. The anatomical structures are described in more detail in this textbook or the other literature used by anatomists, as compared with the references that are mainly used by pain physicians. Moreover, there are differences in experience and perspective in both fields of expertise (Fig. 1).

Studies that incorporate the convergence of clinicians and anatomists' views in approaches to specific anatomical structures may provide more diverse background (c) This is an open-access article distributed under the terms of the Creative Commons Attribution Non-Commercial License (http://creativecommons.org/licenses/by-nc/4.0/), which permits unrestricted non-commercial use, distribution, and reproduction in any medium, provided the original work is properly cited.

(c) The Korean Pain Society, 2021
Author contributions: Yeon-Dong Kim: Writing/manuscript preparation. 


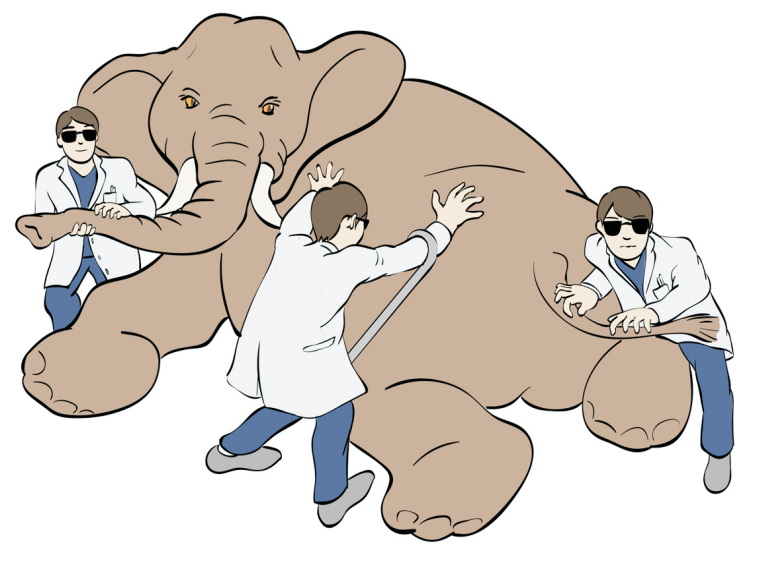

Fig. 1. Different insights on anatomic structures.

knowledge for the effective management of pain.

From a clinical perspective, pain may be caused by overuse, postural changes, degenerative changes, trauma, and surgery. On the other hand, mechanical stimuli such as unexpected entrapment, compression, twisting, and penetration of the nerve caused by variations in structures such as bones, muscles and ligaments can be considered causes of pain from an anatomical perspective. Diagnostic methods, including various imaging modalities, are used to determine the exact relationships when such causes are involved; however, these methods are limited.

The clinical diagnosis of pain is dependent upon a high index of suspicion and physical exam. Finally, the nerve blocks that provide pain relief are the sine qua non for establishing this diagnosis [10]. Interventional pain treatment modalities ranging from nerve blocks to minimally invasive surgery are now used for the diagnosis and treatment of various pain syndromes. Identifying the patient's pain response by precise injections into the desired target based on accurate anatomical knowledge is an essential element of successful pain management.

Pain research is constantly evolving. In the course of this development, ongoing conversations with pain physicians and anatomists will help reduce differences in views on pain-related structures. This will help expand the scope of insight on the anatomical structures by pain physicians, who will gain better clarity in managing patients. Finally, these studies are needed for a better future in pain medicine through the KJP.

\section{CONFLICT OF INTEREST}

No potential conflict of interest relevant to this article was reported.

\section{FUNDING}

No funding to declare.

\section{ORCID}

Yeon-Dong Kim, https://orcid.org/0000-0003-0404-2657

\section{REFERENCES}

1. Manchikanti L, Boswell MV, Raj PP, Racz GB. Evolution of interventional pain management. Pain Physician 2003; 6: 48594.

2. Joo Y, Cho HR, Kim YU. Evaluation of the cross-sectional area of acromion process for shoulder impingement syndrome. Korean J Pain 2020 ; 33: 60-5.

3. Cho H, Kang S, Won HS, Yang M, Kim YD. New insights into pathways of the dorsal scapular nerve and artery for selective dorsal scapular nerve blockade. Korean J Pain 2019; 32: 307-12.

4. Won HS, Jang HY, Moon HS, Zhu PB, Kim YD, Kim H. Fluoroscopic findings of extra-cervical facet joint flow and its incidence on cervical facet joint arthrograms. J Clin Med 2020; 9: 3919.

5. Cankurtaran D, Aykin Yigman Z, Umay E. Factors associated with paravertebral muscle cross-sectional area in patients with chronic low back pain. Korean J Pain 2021; 34: 454-62.

6. Won HS, Iseki M, Hagihira S, Kuk Y, Kim YD, Kim H. Bi-national survey of Korea and Japan related to the injection site for ultrasound-guided stellate ganglion blocks and anatomic comparisons using cadaver dissection. PLoS One 2020; 15: e0232586.

7. Becker RF, Grunt JA. The cervical sympathetic ganglia. Anat Rec 1957; 127: 1-14.

8. Elias M. Cervical sympathetic and stellate ganglion blocks. Pain Physician 2000; 3: 294-304.

9. Malmqvist EL, Bengtsson M, Sörensen J. Efficacy of stellate ganglion block: a clinical study with bupivacaine. Reg Anesth 1992; 17:340-7.

10. Vandam LD, Eckenhoff JE. The anesthesiologist and therapeutic nerve block: technician or physician; with emphasis on the problems of pain relief. Anesthesiology 1954; 15: 8994. 